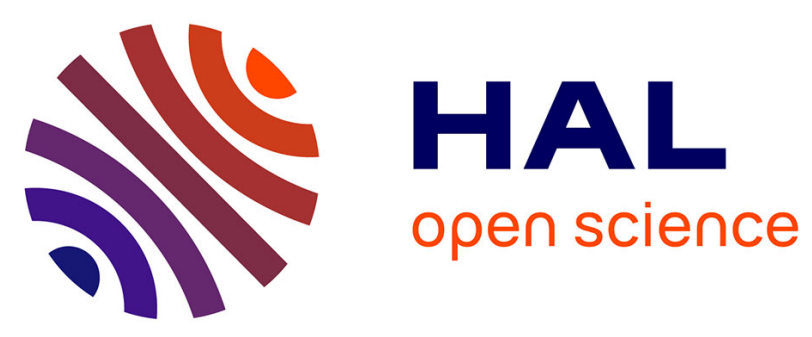

\title{
A Highly Efficient Post-Processing Method for Computing Magnetic Flux in Coils Considering Magnetic and Conductive Regions
}

Limin Huang, Gérard Meunier, Olivier Chadebec, Jean-Michel Guichon, Nicolas Galopin, Brahim Ramdane

\section{To cite this version:}

Limin Huang, Gérard Meunier, Olivier Chadebec, Jean-Michel Guichon, Nicolas Galopin, et al.. A Highly Efficient Post-Processing Method for Computing Magnetic Flux in Coils Considering Magnetic and Conductive Regions. IEEE Transactions on Magnetics, 2018, 58 (3), 10.1109/TMAG.2017.2759255 . hal-02063223

HAL Id: hal-02063223

https://hal.science/hal-02063223

Submitted on 11 Mar 2019

HAL is a multi-disciplinary open access archive for the deposit and dissemination of scientific research documents, whether they are published or not. The documents may come from teaching and research institutions in France or abroad, or from public or private research centers.
L'archive ouverte pluridisciplinaire HAL, est destinée au dépôt et à la diffusion de documents scientifiques de niveau recherche, publiés ou non, émanant des établissements d'enseignement et de recherche français ou étrangers, des laboratoires publics ou privés. 


\title{
A High Efficient Post-Processing Method for Computing Magnetic Flux in Coils Considering Magnetic and Conductive Regions
}

\author{
Limin Huang, Gérard Meunier, Olivier Chadebec, Jean-Michel Guichon, Nicolas Galopin, and Brahim Ramdane \\ Univ. Grenoble Alpes, CNRS, Grenoble INP, G2Elab, 38000 Grenoble, France
}

\begin{abstract}
A high-efficiency post-processing method for computing the magnetic flux in coils is discussed and applied to magnetodynamic problem analysis. The method separates the contribution of magnetic flux in a coil into three parts, respectively generated by source coils, the magnetization of magnetic region and eddy currents in conductive region. The computation of the three components of magnetic flux in coil can be transformed to coil-region-independent integrals, which can help to reduce the mesh size used around the coil, thus increasing the computational efficiency. The method is well adapted to any numerical techniques, such as the finite element and volume integral methods. A typical electromagnetic shielding problem is used to validate the performance of the post-processing magnetic flux computational method at low frequency application.
\end{abstract}

Index Terms-Coil, electromagnetic shielding, magnetic flux, magnetodynamic, volume integral method (VIM).

\section{INTRODUCTION}

$\mathbf{M}$ AGNETIC flux in coils is an important quantity in the analysis, design and optimization of electromagnetic devices. The magnetic flux in a coil is generally calculated in a post-processing step of a computational electromagnetic problem, where the field distribution is firstly obtained by a given numerical method, such as the finite element method (FEM) or the volume integral method (VIM).

With integral formulation method, it is unnecessary to discretize the inactive region. This approach is therefore more suitable for the study of electromagnetic devices with a predominant air region. Thanks to the matrix compression algorithm development, solving electromagnetic problems by integral equations is becoming more and more efficient, from magnetostatic applications [1], [2] to magnetodynamic applications [3], [4] with both magnetic and conductive volume regions, and also a coupling with external circuits.

After solving the problem and getting all the field quantities on the mesh, it is necessary to put forward a high efficient computational method for evaluating magnetic flux in coils in the analysis of electromagnetic devices. In an electromagnetic problem, as illustrated in Fig. 1, the magnetic flux flowing in a coil can be divided into a summation of three terms: 1) one is generated by the magnetic field of source coils; 2) one by the magnetization field of magnetic region and 3) one by the eddy current field of conductive region.

Based on the physical signification of the magnetic flux in coils, these three terms are traditionally calculated by integrating respectively the field quantities on the considered coil region. However, due to the irregular distribution of the fields around the considered coil, the coil mesh should be very fine discretized to have a precise result of the flux

Manuscript received June 26, 2017; revised September 10, 2017; accepted September 26, 2017. Date of publication Month Day, Year; date of current version Month Day, Year. Corresponding author: L. Huang (e-mail: Limin.Huang@g2elab.grenoble-inp.fr).

Color versions of one or more of the figures in this paper are available online at http://ieeexplore.ieee.org.

Digital Object Identifier (inserted by IEEE).

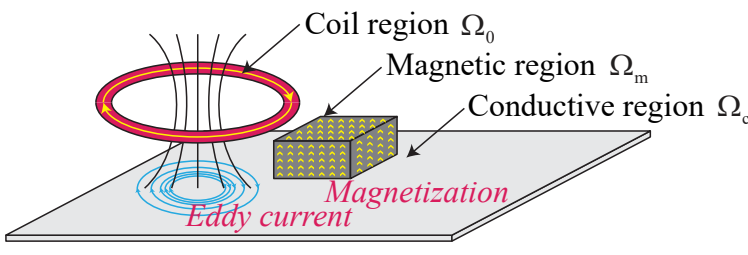

Fig. 1. Illustration of a magnetodynamic problem regions.

computation. The increased element number will also increase the computational complexity of the problem.

To avoid using too complex coil mesh, a coil-regionindependent method has been presented in [5]. This computational method allows obtaining the flux component through a coil by integrating directly on the magnetic region rather than the coil region. It has shown a good efficiency in the study of inductive power transmission system for the mutualinductance calculation. However, this method can only deal with the magnetostatic problems and has not been extended yet to conductive region in magnetodynamic context.

Following the previous research, in this paper we propose a new general approach to compute the magnetic flux in coils under magnetodynamic context. The approach begins by solving a magnetodynamic problem by an integral formulation, then dividing the magnetic flux flowing in a coil into the summation of three flux components, which can be calculated by simplified integrals. Finally, the proposed coil-regionindependent computational method is applied and verified in an electromagnetic shielding application example.

\section{Integral Formulation Method}

A magnetodynamic problem can be described as a problem with three typical regions: 1) the source coil region (noted by $\Omega_{0}$ ), 2) the magnetic region (noted by $\Omega_{\mathrm{m}}$ ) and 3 ) the conductive electrical region (noted by $\Omega_{\mathrm{c}}$ ). Starting from Maxwell equations, the electric field $\mathbf{E}$ and the magnetic field $\mathbf{H}$ can be expressed by the potential formulation, as

$$
\left\{\begin{array}{l}
\mathbf{E}=-\frac{\mathrm{d} \mathbf{A}}{\mathrm{d} t}-\operatorname{grad} V \\
\mathbf{H}=\mathbf{T}-\operatorname{grad} \phi
\end{array}\right.
$$


where $\mathbf{T}$ and $\mathbf{A}$ are the electric and magnetic vector potentials, $V$ and $\phi$ are the electric and magnetic scalar potentials.

\section{A. Magnetodynamic Integral Equation}

Displacement currents can be neglected when considering the low frequency magnetodynamic problem and application. According to the adequate gauges, all the four potentials can be expressed from the integral formulation that are limited to the regions $\Omega_{0}, \Omega_{\mathrm{m}}$ and $\Omega_{\mathrm{c}}$, as

$$
\left\{\begin{aligned}
\mathbf{A}_{p} & =\frac{\mu_{0}}{4 \pi}\left(\int_{\Omega_{0}} \frac{\mathbf{J}_{0 q}}{r} \mathrm{~d} \Omega+\int_{\Omega_{\mathrm{m}}} \frac{\mathbf{M}_{q} \times \mathbf{r}}{r^{3}} \mathrm{~d} \Omega+\int_{\Omega_{\mathrm{c}}} \frac{\mathbf{J}_{q}}{r} \mathrm{~d} \Omega\right) \\
\frac{\mathrm{d} V_{p}}{\mathrm{~d} t} & =\frac{1}{4 \pi \varepsilon_{0}} \int_{\Omega_{\mathrm{c}}} \frac{\mathbf{J}_{q} \cdot \mathbf{r}}{r^{3}} \mathrm{~d} \Omega \\
\mathbf{T}_{p} & =\frac{1}{4 \pi}\left(\int_{\Omega_{0}} \frac{\mathbf{J}_{0 q} \times \mathbf{r}}{r^{3}} \mathrm{~d} \Omega+\int_{\Omega_{\mathrm{c}}} \frac{\mathbf{J}_{q} \times \mathbf{r}}{r^{3}} \mathrm{~d} \Omega\right) \\
\Phi_{p} & =\frac{1}{4 \pi} \int_{\Omega_{\mathrm{m}}} \frac{\mathbf{M}_{q} \cdot \mathbf{r}}{r^{3}} \mathrm{~d} \Omega
\end{aligned}\right.
$$

The formulation allows computing exact solution of fields $\mathbf{E}$ and $\mathbf{H}$ at any point $p$ from the knowledge of a point $q$ (current densities $\mathbf{J}_{0}$ in the coil region, the magnetization field $\mathbf{M}$ in magnetic region and $\mathbf{J}$ in conductive region). Because $\mathbf{M}$ and $\mathbf{J}$ are also variables to be solved, constitutive laws of materials should be added to obtain a complete solution. In the magnetodynamic context, two constitutive laws which link, respectively, the current density $\mathbf{J}$ to the electric field $\mathbf{E}$ (in $\Omega_{\mathrm{c}}$ ) and the magnetization $\mathbf{M}$ to the magnetic field $\mathbf{H}$ (in $\Omega_{\mathrm{m}}$ ) should be essentially considered as

$$
\left\{\begin{aligned}
\mathbf{J} & =\sigma \mathbf{E} \\
\mathbf{M} & =\chi \mathbf{H}=\left(v_{0}-v\right) \mathbf{B}
\end{aligned}\right.
$$

\section{B. Facet Interpolation}

The magnetic region and the conductive region can be discretized with Whitney first-order facet finite element functions. In this case, the interpolation of current and flux density are

$$
\left\{\begin{array}{l}
\mathbf{J}=\sum_{j} \mathbf{w}_{j} I_{j} \\
\mathbf{B}=\sum_{g} \mathbf{w}_{g} \psi_{g}
\end{array}\right.
$$

where $\mathbf{w}_{j}$ and $\mathbf{w}_{g}$ are facet shape functions; $I_{j}$ and $\psi_{g}$ are the current or flux across the facets. Facet interpolation is well adapted to unknowns $\mathbf{J}$ and $\mathbf{B}$ since it imposes the continuity of the flux into the finite element mesh [4].

Then, two Galerkin procedures can be then associated respectively in the magnetic region $\Omega_{\mathrm{m}}$ and the conductive region $\Omega_{\mathrm{c}}$ :

$$
\left\{\begin{aligned}
\int_{\Omega_{\mathrm{c}}} \mathbf{w}_{i}\left(\frac{\mathbf{J}}{\sigma}-\mathbf{E}\right) \mathrm{d} \Omega & =0 \\
\int_{\Omega_{\mathrm{m}}} \mathbf{w}_{f}\left(\frac{\mathbf{M}}{\chi}-\mathbf{H}\right) \mathrm{d} \Omega & =0
\end{aligned}\right.
$$

where the field distribution $\mathbf{E}$ and $\mathbf{H}$ are obtained thanks to (1) and (2). By using facet interpolations, facet shape functions are chosen for the Galerkin projection of $\mathbf{J}$ and $\mathbf{B}$. Furthermore, such a method can also lead to an equivalent circuit representation which avoids the convexity problem and strongly imposes the solenoidality of $\mathbf{J}$ and $\mathbf{B}$ [3].

\section{Matrix System Computation}

From relations (2) and the constitutive laws, the relation between $\mathbf{J}, \mathbf{A}, \mathbf{B}$ and $\mathbf{T}$ can be expressed in terms of currents $I_{j}$ through the facets of the conductive region and magnetic flux $\Psi_{j}$ across the facets of magnetic region. After the Galerkin procedures in the magnetic region $\Omega_{\mathrm{m}}$ and the conductive region $\Omega_{\mathrm{c}}$, a matrix system of equations, which links the difference of averaged potentials between two adjacent elements $(V$ and $\Phi)$ with the flux through facets $\left(I_{j}\right.$ and $\Psi_{j}$ ) is obtained as

$$
\left[\begin{array}{cc}
{[\mathbf{R}]+\frac{\mathrm{d}}{\mathrm{d} t}[\mathbf{L}]} & \frac{\mathrm{d}}{\mathrm{d} t}[\mathbf{C}] \\
{[\mathbf{D}]} & {[\mathbf{E}]}
\end{array}\right]\left\{\begin{array}{c}
I \\
\Psi
\end{array}\right\}=\left\{\begin{array}{c}
\Delta V \\
\Delta \Phi
\end{array}\right\}+\left\{\begin{array}{c}
U \\
Q
\end{array}\right\}
$$

where the coefficients of the matrix is defined as

$$
\left\{\begin{array}{l}
\mathbf{R}_{i j}=\int_{\Omega_{\mathrm{c}}} \mathbf{w}_{i} \cdot \frac{\mathbf{w}_{j}}{\sigma} \mathrm{d} \Omega \quad \mathbf{L}_{i j}=\frac{\mu_{0}}{4 \pi} \int_{\Omega_{\mathrm{c}}} \mathbf{w}_{i} \cdot \int_{\Omega_{\mathrm{c}}} \frac{\mathbf{w}_{j}}{r} \mathrm{~d} \Omega \mathrm{d} \Omega \\
\mathbf{C}_{i g}=\frac{\mu_{0}}{4 \pi} \int_{\Omega_{\mathrm{c}}} \mathbf{w}_{i} \cdot \int_{\Omega_{\mathrm{m}}}\left(\nu_{0}-\nu\right) \frac{\mathbf{w}_{g} \times \mathbf{r}}{r^{3}} \mathrm{~d} \Omega \mathrm{d} \Omega \\
\mathbf{D}_{f i}=-\frac{1}{4 \pi} \int_{\Omega_{\mathrm{c}}} \mathbf{w}_{f} \cdot \int_{\Omega_{\mathrm{m}}} \frac{\mathbf{w}_{j} \times \mathbf{r}}{r^{3}} \mathrm{~d} \Omega \mathrm{d} \Omega \\
\mathbf{E}_{f g}=\int_{\Omega_{\mathrm{m}}} \nu \mathbf{w}_{f} \cdot \mathbf{w}_{g} \mathrm{~d} \Omega \\
\mathbf{F}_{f g}=\frac{1}{4 \pi} \int_{\Gamma_{f}} \frac{1}{S_{f}} \cdot \int_{\Omega_{\mathrm{m}}}\left(\nu-\nu_{0}\right) \frac{\mathbf{w}_{g} \cdot \mathbf{r}}{r^{3}} \mathrm{~d} \Omega \mathrm{d} \Omega
\end{array}\right.
$$

and the terms $U$ and $Q$ in the second hand are the source terms produced by all the active coils in the problem. Such a matrix system can be easily calculated by magnetic vector potential formulation that combines equivalent network based on Whitney facet interpolation and Green's function volume integral method proposed in [4].

\section{Magnetic Flux Computation}

Formulated by the magnetic vector potential $\mathbf{A}$, the magnetic flux $\Phi$ through a considered coil $k$, with a region denoted by $\Omega_{0_{k}}$, can be expressed by an integral on the coil region:

$$
\Phi_{k}=\int_{\Omega_{0_{k}}} \mathbf{j}_{0_{k}} \cdot \mathbf{A} \mathrm{d} \Omega
$$

where $\mathbf{j}_{0_{k}}$ is a vector function describing the normalized current density of the considered coil region $\Omega_{0_{k}}$. For a magnetodynamic problem containing three typical domains: 1) source coil region $\Omega_{0_{k}} ; 2$ ) non-conducting magnetic region $\Omega_{\mathrm{m}}$ and 3) electrical conductive regions $\Omega_{\mathrm{c}}$, as illustrated in Fig. 1, the magnetic vector potential $\mathbf{A}$ can be separated in three components, i.e. $\mathbf{A}=\mathbf{A}_{0}+\mathbf{A}_{\mathrm{m}}+\mathbf{A}_{\mathrm{c}}$, where $\mathbf{A}_{0}, \mathbf{A}_{\mathrm{m}}$ and $\mathbf{A}_{\mathrm{c}}$ are generated respectively by the three aforementioned regions. Therefore, the contribution of total magnetic flux $\Phi_{k}$ through a coil $k$ can be expressed by

$$
\Phi_{k}=\Phi_{0_{k}}+\Phi_{\mathrm{m}_{k}}+\Phi_{\mathrm{c}_{k}}
$$


where $\Phi_{0_{k}}$ is the contribution of all source coils in vacuum, $\Phi_{\mathrm{m}_{k}}$ is the contribution of the magnetization of magnetic region and $\Phi_{c_{k}}$ is the contribution of eddy currents in conductive region.

\section{A. $\Phi_{0_{k}}$ Computation}

In a magnetodynamic problem with multiple coils, the magnetic vector potential $\mathbf{A}_{0_{k}}$ through the coil $k$ can be computed by considering all the coils in vacuum, as

$$
\mathbf{A}_{0_{k}}=\frac{\mu_{0}}{4 \pi} \sum_{l}\left(\int_{\Omega_{0_{l}}} \frac{\mathbf{j}_{0_{l}}}{r} \mathrm{~d} \Omega\right) I_{l}
$$

where each coil $l$ is defined with a region $\Omega_{0_{l}}$ and carrying a current $I_{l}$. Thus, the first part of flux $\Phi_{0_{k}}$, generated by all coils is the problem, can be calculated by a double integral:

$$
\Phi_{0_{k}}=\frac{\mu_{0}}{4 \pi} \int_{\Omega_{0_{k}}} \mathbf{j}_{0_{k}} \cdot\left(\sum_{l} I_{l} \int_{\Omega_{0_{l}}} \frac{\mathbf{j}_{0_{l}}}{r} \mathrm{~d} \Omega\right) \mathrm{d} \Omega
$$

The double integration (11) can be computed by a semianalytical integration method. An analytical expression proposed in [6] can be used to evaluate the first integral on the source coil region $\Omega_{0_{l}}$, and the quadratic Gauss points integration can be used to compute the first integral on the objective coil region $\Omega_{0_{k}}$.

\section{B. $\Phi_{\mathrm{m}_{k}}$ Computation}

Considering the magnetization effect in the magnetic region $\Omega_{\mathrm{m}}$, the magnetic vector potential $\mathbf{A}_{\mathrm{m}}$ can be computed as an integral on $\Omega_{\mathrm{m}}$. Similarly, the flux component $\Phi_{\mathrm{m}_{k}}$ through the coil $k$ can be computed by a double integral:

$$
\left\{\begin{aligned}
\mathbf{A}_{\mathrm{m}} & =\frac{\mu_{0}}{4 \pi} \int_{\Omega_{\mathrm{m}}} \mathbf{M} \times \nabla\left(\frac{1}{r}\right) \mathrm{d} \Omega_{\mathrm{m}} \\
\Phi_{\mathrm{m}_{k}} & =\frac{\mu_{0}}{4 \pi} \int_{\Omega_{0_{k}}} \mathbf{j}_{0_{k}}\left[\int_{\Omega_{\mathrm{m}}} \mathbf{M} \times \nabla\left(\frac{1}{r}\right) \mathrm{d} \Omega_{\mathrm{m}}\right] \mathrm{d} \Omega
\end{aligned}\right.
$$

Because the computation of the magnetic flux component $\Phi_{\mathrm{m}_{k}}$ is related to the considered coil region $\Omega_{0_{k}}$, it should thus be sufficiently meshed to have a precise result. A great number of elements will increase dramatically the computation time, it is relatively inefficient to solve directly the double integral, especially dealing with the complex geometries.

A method which allows to obtain $\Phi_{\mathrm{m}_{k}}$ by integrating directly in the magnetic region $\Omega_{\mathrm{m}}$ has been presented in [5]. This method begins with the usage of the partial integral theorem to the equation (8), as

$$
\begin{aligned}
\Phi_{\mathrm{m}_{k}} & =\int_{\Omega_{0_{k}}} \mathbf{j}_{0_{k}} \cdot \mathbf{A}_{\mathrm{m}} \mathrm{d} \Omega=\int_{\Omega}\left(\nabla \times \mathbf{h}_{0_{k}}\right) \cdot \mathbf{A}_{\mathrm{m}} \mathrm{d} \Omega \\
& =\int_{\Omega} \mathbf{h}_{0_{k}} \cdot\left(\nabla \times \mathbf{A}_{\mathrm{m}}\right) \mathrm{d} \Omega
\end{aligned}
$$

where $\mathbf{h}_{0_{k}}$ represents the normalized magnetic field generated by the coil $k$ with $1 \mathrm{~A}$. Considering the relation $\nabla \times \mathbf{A}_{\mathrm{m}}=$ $\mu_{0}\left(-\nabla \varphi_{\mathrm{r}}+\mathbf{M}\right)$, the equation (13) becomes

$$
\Phi_{\mathrm{m}_{k}}=\mu_{0} \int_{\Omega} \mathbf{h}_{0_{k}}\left(-\nabla \varphi_{\mathrm{r}}+\mathbf{M}\right) \mathrm{d} \Omega
$$

where the integral $\int_{\Omega} \mathbf{h}_{0_{k}}\left(\nabla \varphi_{\mathrm{r}}\right) \mathrm{d} \Omega$ can be proved to be 0 by applying another time the partial integration theorem and considering the boundary condition of infinity. Finally, the magnetic flux component $\Phi_{\mathrm{m}_{k}}$ generated by the magnetization effect can be computed as

$$
\Phi_{\mathrm{m}_{k}}=\mu_{0} \int_{\Omega_{\mathrm{m}}} \mathbf{h}_{0_{k}} \cdot \mathbf{M d} \Omega
$$

\section{C. $\Phi_{\mathrm{c}_{k}}$ Computation}

Similarly, by considering the eddy currents effect in the conductive region $\Omega_{\mathrm{c}}$, the magnetic vector potential $\mathbf{A}_{\mathrm{c}}$ can be computed as an integral on $\Omega_{\mathrm{c}}$ and the flux component $\Phi_{\mathrm{c}_{\mathrm{k}}}$ through the coil $k$ can be computed as a double integral:

$$
\left\{\begin{aligned}
\mathbf{A}_{\mathrm{c}} & =\frac{\mu_{0}}{4 \pi} \int_{\Omega_{\mathrm{c}}} \frac{\mathbf{J}}{r} \mathrm{~d} \Omega_{\mathrm{c}} \\
\Phi_{\mathrm{c}_{k}} & =\frac{\mu_{0}}{4 \pi} \int_{\Omega_{0_{k}}} \mathbf{j}_{0_{k}}\left[\int_{\Omega_{\mathrm{c}}} \frac{\mathbf{J}}{r} \mathrm{~d} \Omega_{\mathrm{c}}\right] \mathrm{d} \Omega
\end{aligned}\right.
$$

In order to increase the computational efficiency by solving directly the double integral in (16) for the flux component $\Phi_{\mathrm{c}_{\mathrm{k}}}$, here we propose a coil-region-independent method which allows to simplify the double integral to a single integration in the conductive region $\Omega_{\mathrm{c}}$. The method begins by applying the partial integral theorem:

$$
\begin{aligned}
\Phi_{\mathrm{c}_{k}} & =\int_{\Omega_{0_{k}}} \mathbf{j}_{0_{k}} \cdot \mathbf{A}_{\mathrm{c}} \mathrm{d} \Omega=\int_{\Omega}\left(\nabla \times \mathbf{h}_{0_{k}}\right) \cdot \mathbf{A}_{\mathrm{c}} \mathrm{d} \Omega \\
& =\int_{\Omega} \nabla \cdot\left(\mathbf{h}_{0_{k}} \times \mathbf{A}_{\mathrm{c}}\right) \mathrm{d} \Omega-\int_{\Omega} \mathbf{h}_{0_{k}} \cdot\left(\nabla \times \mathbf{A}_{\mathrm{c}}\right) \mathrm{d} \Omega
\end{aligned}
$$

where the term $\nabla \cdot\left(\mathbf{h}_{0_{k}} \times \mathbf{A}_{\mathrm{c}}\right)=0$. The magnetic flux $\Phi_{\mathrm{c}_{k}}$ generated by eddy currents can be then computed by

$$
\Phi_{\mathrm{c}_{k}}=\int_{\Omega} \mathbf{h}_{0_{k}} \cdot\left(\nabla \times \mathbf{A}_{\mathrm{c}}\right) \mathrm{d} \Omega
$$

In the integral (18), the terms $\mathbf{h}_{0_{k}}$ and $\nabla \times \mathbf{A}_{\mathrm{c}}$ can be substituted by:

$$
\left\{\begin{array}{l}
\mathbf{h}_{0_{k}}=\frac{1}{\mu_{0}}\left(\nabla \times \mathbf{a}_{0_{k}}\right) \\
\nabla \times \mathbf{A}_{\mathrm{c}}=\mu_{0} \mathbf{H}_{\mathrm{c}}
\end{array}\right.
$$

where $\mathbf{H}_{\mathrm{c}}$ is the field produce by the eddy current. Then (18) becomes

$$
\Phi_{c_{k}}=\int_{\Omega} \frac{1}{\mu_{0}}\left(\nabla \times \mathbf{a}_{0_{k}}\right) \cdot\left(\mu_{0} \mathbf{H}_{\mathrm{c}}\right) \mathrm{d} \Omega
$$

By applying another time the partial integration theorem, the equation (20) becomes

$$
\Phi_{\mathrm{c}_{k}}=\int_{\Omega} \mathbf{a}_{0_{k}} \cdot\left(\nabla \times \mathbf{H}_{\mathrm{c}}\right) \mathrm{d} \Omega+\int_{\Omega} \nabla \cdot\left(\mathbf{a}_{0_{k}} \times \mathbf{H}_{\mathrm{c}}\right) \mathrm{d} \Omega
$$

According to the divergence theorem, the second term $\int_{\Omega} \nabla \cdot\left(\mathbf{a}_{0_{k}} \times \mathbf{H}_{\mathrm{c}}\right) \mathrm{d} \Omega$ becomes $\int_{\Gamma} \mathbf{a}_{0_{k}} \times \mathbf{H}_{\mathrm{c}} \mathrm{d} \Gamma$. Because of the continuity, this term can be expended to the infinity boundary $\Gamma_{\infty}$, where the field is null, and so the integral $\int_{\Omega} \nabla \cdot\left(\mathbf{a}_{0_{k}} \times \mathbf{H}_{\mathrm{c}}\right) \mathrm{d} \Omega=0$, thus

$$
\Phi_{\mathrm{c}_{k}}=\int_{\Omega} \mathbf{a}_{0_{k}} \cdot\left(\nabla \times \mathbf{H}_{\mathrm{c}}\right) \mathrm{d} \Omega
$$




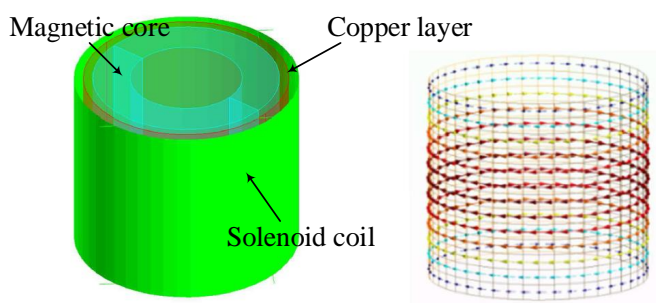

Fig. 2. 3-D axisymmetric electromagnetic shielding application example. (a) Geometry and (b) eddy currents density on the conductive region.

In the conductive region $\Omega_{\mathrm{c}}$, the relation between $\mathbf{H}_{\mathrm{c}}$ and $\mathbf{J}_{\mathrm{c}}$ can be written as

$$
\nabla \times \mathbf{H}_{\mathrm{c}}=\mathbf{J}_{\mathrm{c}}
$$

By substituting the relation (23) into (22), the integral of computing the flux component $\Phi_{\mathrm{c}_{k}}$ becomes finally an integral on the conductive region $\Omega_{\mathrm{c}}$ :

$$
\Phi_{\mathrm{c}_{k}}=\int_{\Omega} \mathbf{a}_{0_{k}} \cdot\left(\nabla \times \mathbf{H}_{\mathrm{c}}\right) \mathrm{d} \Omega=\int_{\Omega_{\mathrm{c}}} \mathbf{a}_{0_{k}} \cdot \mathbf{J}_{\mathrm{c}} \mathrm{d} \Omega
$$

where $\mathbf{a}_{0_{k}}$ represents the normalized magnetic vector potential generated by the coil $k$ with $1 \mathrm{~A}$, and $\mathbf{J}_{\mathrm{c}}$ means the eddy current density. The computation of the magnetic flux $\Phi_{\mathrm{c}_{k}}$, which is generated by eddy current is finally changed into an integral which is related only to the domain of electrical conductive region $\Omega_{\mathrm{c}}$.

\section{Application Example}

The proposed coil-region-independent computational method for magnetic flux is tested by a strong magneticelectric coupling problem, as illustrated in Fig. 2. The test 3-D problem is composed of:

1) solenoid coil (radius $6 \mathrm{~mm}$, thickness $0.1 \mathrm{~mm}$, height $10 \mathrm{~mm}$, effective value of current: 1 Ampere, number of turns: 100);

2) linear magnetic core (average radius $4 \mathrm{~mm}$, thickness $2 \mathrm{~mm}$, height $10 \mathrm{~mm}$, relative permeability 100);

3) thin surface copper layer (radius $5.5 \mathrm{~mm}$, thickness $0.1 \mathrm{~mm}$, height $10 \mathrm{~mm}$, conductivity $55 \times 10^{6} \mathrm{~S} / \mathrm{m}$ ).

After modeling the probem in 3-D context, the problem is solved by the volume integral method (VIM) to obtain the fields distribution. The flux is computed by summing the three components at the post-processing step.

Thanks to the advantage of VIM and the proposed magnetic flux computation method, only the active regions (magnetic core structures and the surface copper layer) should be fine considered and discretized. Only one tenth of the element number in the active region can thus realize a precision of $0.5 \%$ in comparison with the FEM which should take account of the predominant air region and the traditional magnetic flux computational method where the coils should be fine meshed.

Figure 3 shows the variation of the flux in the coil versus the frequency, with an axisymmetric finite element analysis to the same problem in the software Flux as reference. The comparison to the results with a finite element method shows a difference less than $0.4 \%$ at any frequency.

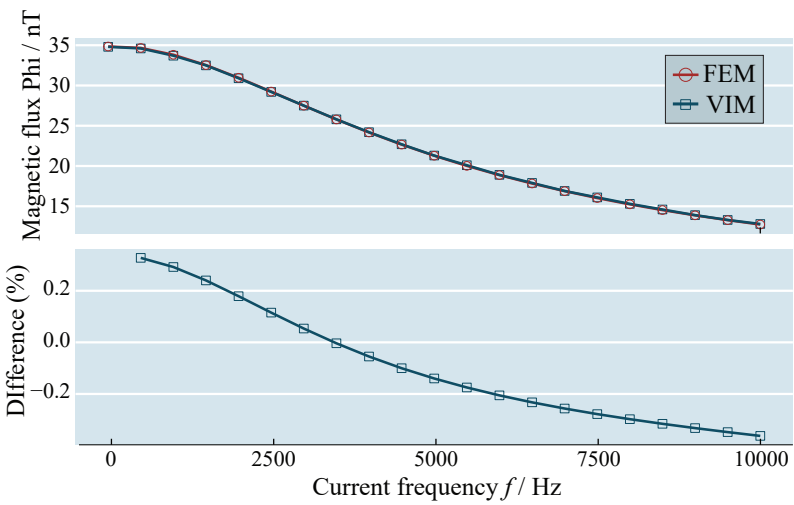

Fig. 3. Comparison between the proposed post-processing method results and FEM simulation results of magnetic flux in the coil.

In this configuration, the thin surface copper layer is placed between the coil and the magnetic core, to realize an electromagnetic shield. The computational results of the flux in the coil versus the frequency shows that when the system operation frequency increases, the eddy currents on the thin surface copper begin to act as a shield and the magnetic flux in coil decreases.

\section{CONCLUSION}

A coil-region-independent post-processing computational method for the magnetic flux in coils under magnetodynamic context is presented and applied to solve electromagnetic shielding problem. The computational method begins by solving the magnetodynamic problem with integral formulation method, which is unnecessary to consider the massive inactive region and it can thus ignore the predominant air region. Then, a high efficient computational method for magnetic flux has been presented by using coil-region-independent integrals. The decomposition of the magnetic flux in coils considering both magnetic and conductive regions is well adapted to the magnetodynamic problem. The method to compute the magnetic flux in coil at post-processing step by three simple integrals is general and it can be applied to any numerical methods, such as FEM and VIM.

\section{REFERENCES}

[1] A. Canova and M. Repetto, "Integral solution of nonlinear magnetostatic field problems," IEEE Trans. Magn., vol. 37, no. 3, pp. 1070-1077, May 2001.

[2] A. Morandi, M. Fabbri and P. L. Ribani, "A modified formulation of the volume integral equations method for 3-D magnetostatics," IEEE Trans. Magn., vol. 46, no. 11, pp. 3848?3859, Nov. 2010.

[3] G. Meunier, O. Chadebec and J. M. Guichon, "A magnetic flux-electric current volume integral formulation based on facet elements for solving electromagnetic problems," IEEE Trans. Magn., vol. 51, no. 3, pp. 1-4, Mar. 2015.

[4] G. Meunier, O. Chadebec, J. M. Guichon, V. Le-Van, J. Siau, B. Bannwarth and F. Sirois, "AT volume integral formulations for solving electromagnetic problems in the frequency domain," IEEE Trans. Magn., vol. 52, no. 3, pp. 1-4, Mar. 2016.

[5] L. Huang, G. Meunier, O. Chadebec, J. M. Guichon, Y. Li and Z. He "General Integral Formulation of Magnetic Flux Computation and its Application in Inductive Power Transfer System," IEEE Trans. Magn., vol. 53, no. 6, pp. 1-4, June 2017.

[6] M. Fabbri, "Magnetic flux density and vector potential of uniform polyhedral sources," IEEE Trans. Magn., vol. 44, no. 1, pp. 32-36, Jan. 2008 